\title{
CAN A QUARK SHELL MODEL LEAD TO NUCLEON CLUSTERS?
}

\author{
Y. SUZUKI ' ${ }^{\mathrm{I}}$ and K.T. HECHT \\ Physics Department, University of Michigan ${ }^{2}$, Ann Arbor, MI 48109, USA
}

Received 13 June 1989; revised manuscript reccived 17 October 1989

\begin{abstract}
The six-quark deuteron system is examined within the framework of the Bonn quark shell model to see whether this model can lead to a quark density distribution indicative of two spatially scparated threc-quark clusters. The deuteron ground state always lcads to a quark density distribution with a centrai maximum. The configuration mixing of the model does not furnish the needed strong spatial correlation into three-quark clusters.
\end{abstract}

Recently [1-3] it has been suggested that the shell model of the nucleus can be understood in terms of a shell model of $3 A$ quarks moving independently in an MIT-type bag of nucleus size rather than the conventional model based on the independent motion of $A$ nuclcons. This "Bonn" model seems to give a very natural explanation of the Mayer-Jensen level sequence in terms of relativistic quark dynamics. It also contains the nuclcar radius law $R=r_{0} A^{1 / 3}$ through the equilibrium condition betwcen the inner pressure from quarks in occupied single particle states and the outer vacuum pressure of MIT bag type with a bag constant $B$ which is independent of nucleon number and not too far off the MIT bag value. This quark model of the nucleus is based on the idea that each color singlet three-quark substructure in the low cncrgy states of a nucleus contains one quark pair coupled to $J=0, T=0$ in its required color $\overline{3} \mathrm{SU}(3)$-(01) state. Such a pair is therefore spectroscopically incrt, and the $J, T$ structure of an open shell nucleus is determined by the $A$ quarks not in $J=0, T=0$ coupled pairs, in particular those in an unfilled $j$-subshell. A central feature of this model is a strong $J=0, T=0$ pairing interaction which separates non-nucleonic excitations, of $\Delta$-type e.g., from the conventional nuclear states with the same quantum numbers as states built from nucleons in the same j-orbits.

\footnotetext{
1 Permanent address: Physics Department, Niigata University, Niigata 950-21, Japan.

2 Supported in part by the National Science Foundation.
}

This "Bonn" shell model based on quarks is also motivated very much by the fact that individual nucleon three-quark structures of MIT bag character and size $(\sim 1 \mathrm{fm})$ take up an appreciable fraction of the total nuclear volume. Such three-quark bags would therefore be moving through each other, merging, and fusing into possibly larger structures a large fraction of the time. The question therefore arises: Does a model based on $3 A$ quarks moving in a single bag give an acceptable alternative for a zeroth order description of a heavy nuclcus? In particular, does such a $3 \mathrm{~A}$ quark wave function have a large overlap with a cluster wave function built from $A$ three-quark bags if antisymmetrization between quarks in different bags is properly taken into account?

We are reminded of a similar question from the history of nuclear models: Are nuclei such as ${ }^{8} \mathrm{Be},{ }^{12} \mathrm{C}$, ${ }^{16} \mathrm{O},{ }^{20} \mathrm{Ne}$, and even heavier nuclei described best in terms of alpha-cluster models or in terms of the conventional shell model? In this case it is well known that there is a large overlap between the shell model wave function and the appropriatcly antisymmetrized alpha cluster wave function [4], particularly if sufficient configuration mixing is included in the shell model description.

Recently, Talmi [5] has criticized the Bonn shell model based on quarks, by noting that the single $j$ shell three-quark wave function leads to very small single-nucleon pickup spectroscopic factors. We are, however, again reminded of the fact that early simple shell model calculations of alpha-spectroscopic fac- 
tors were too small by many orders of magnitude and that the most sophisticated present-day configuration-mixed calculation leads to a predicted alpha spectroscopic factor in ${ }^{212}$ Po which is still too small [6] by a factor of $\sim 10$.

The Bonn shell model of the nucleus based on quarks does lead to strong configuration mixing. The key question remains: Can this configuration mixing begin to develop the strong spatial correlations into three-quark clusters which are required by the experimental facts. If such a spatial correlation could be demonstrated, the conventional shell model picture of $A$ nucleons and the shell of $3 A$ quarks may simply be alternate zeroth order descriptions of the same basic structure.

To investigate this question it may be sufficient and in fact crucial to look at the simplest complex nucleus, viz. the deuteron. The deuteron is indisputably a system of two nucleons, two relatively well-separated three-quark clusters, and not a compact system of six quarks. In the analogous "two-cluster" system of the alpha particle model, ${ }^{8} \mathrm{Be}$, the experimental facts are again indisputable: ${ }^{8} \mathrm{Be}$ is two alpha particles. However, here it is well known that even the simplest intrinsic $\mathrm{s}^{4} \mathrm{p}_{z}^{4}$ shell model wave function of ${ }^{8} \mathrm{Be}$ leads to two peaks in the density distribution of nucleons with a central minimum. These peaks correspond to the two alpha particles. This is shown in fig. 1. Morcover, it is known that the two-alpha clus-

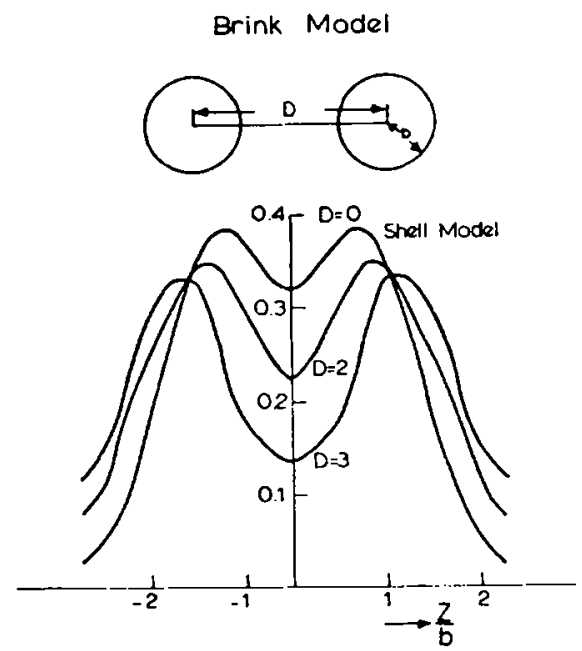

Fig. 1. The nuclcon density distribution for ${ }^{8} \mathrm{Be}$ along the $z$-axis, $b=1.31 \mathrm{fm}$. See ref. [7]. ter wave functions with larger alpha-alpha separation have an improved overlap with a shell model wave function with strong configuration mixing. The crucial question for the Bonn shell model of the nucleus based on quarks is the following: Docs the full implementation of the Bonn model begin to show a comparable separation into two three-quark nucleons if we examine the density distribution of quarks in the six-quark system?

The Bonn shell model of quarks is based on the hamiltonian

$H=\sum_{j} \epsilon_{j} N_{j}-g \sum_{j 1 j_{2}} \sum_{i<k} A^{\dagger}\left(j_{1}\right)_{i k} A\left(j_{2}\right)_{i k}$,

where $\epsilon_{j}$ is the single-quark encrgy in a spherical MIT bag with sharp boundary at $r=R$ (mass term $\rightarrow \infty$ at $r=R$ ). We choose $R=2.5 \mathrm{fm}$ for the six-quark system. Calculations were carried out both for zero rest mass quarks, $m=0$, and for $m=300 \mathrm{MeV}$. The operators are $N_{j}$, the quark number operator, and $A^{\prime}\left(j_{1}\right)_{i k}$, the quark $J=0, T=0$, color antitriplet pair-creation operator for the quark orbit $j_{1}$,

$$
\begin{aligned}
& A^{\dagger}\left(j_{1}\right)_{i k} \\
& \quad=\sum_{m} \sum_{m t}(-1)^{j_{1}-m+1 / 2-m_{t}} A_{j ! m m_{t} i}^{\dagger} a_{j_{1}-m-m, k}^{\dagger},
\end{aligned}
$$

where $i, k=1,2,3$ are color indices. The strength of the pairing term was set at $g=6.3 \mathrm{MeV}$ (for $m=0$ ) and $g=5.2 \mathrm{MeV}$ (for $m=300$ ) so that the six-quark state corresponding to two $\Delta$ excitations in the pure $0 \mathrm{~s}_{1 / 2}$ orbit is at an excitation of $500-600 \mathrm{MeV}$ above the deuteron ground state. These $g$ 's agree well with calculations for the three-quark system where a single $\left(0 \mathrm{~s}_{1 / 2}\right)^{3} \Delta$ excitation is placed at $293 \mathrm{MeV}$ above the $j=1 / 2, T=1 / 2$ nucleon ground state. The model space was chosen to include the 12 lowest quark orbits, viz. $0 \mathrm{~s}_{1 / 2}, 0 \mathrm{p}_{3 / 2}, 0 \mathrm{p}_{1 / 2}, 0 \mathrm{~d}_{5 / 2}, 0 \mathrm{~d}_{3 / 2}, 1 \mathrm{~s}_{1 / 2}, 0 \mathrm{f}_{7 / 2}$, $0 f_{5 / 2}, 0 g_{9 / 2}, 1 p_{3 / 2}, 1 p_{1 / 2}, 1 p_{1 / 2}, 0 g_{7 / 2}$. For mixed configuration calculations an $\mathrm{SO}(6) \supset U(3)$ algebra is very useful [8]. State normalization factors can be found in ref. [8]. However, the single particle term in the hamiltonian breaks the $\mathrm{SO}(6)$ symmetry and, contrary to some of the calculations of ref. [3], this is fully taken into account. The deuteron ground state is made up of one quark pair in the $0 \mathrm{~s}_{1 / 2}$ orbit coupled to $J=1, T=0, \operatorname{SU}(3)$-color (20) symmetry, (the "intrinsic" state of ref. [8] with $[\sigma]=[2])$, with two additional $J=0,7=0$ quark pairs distributed among 
the 12 listed single quark orbits. This leads to a 78component six-quark state with extreme configuration mixing

$$
\begin{aligned}
& \sum_{j_{1, j 2}} c\left(j_{1}, j_{2}\right) \frac{1}{K\left(j_{1}, j_{2}\right)} \\
& \quad \times\left[\left[A^{\dagger}\left(j_{1}\right) \times A^{\dagger}\left(j_{2}\right)\right]\right\}_{=0,}^{02} T=0 \\
& \left.\left.\quad \times\left[a_{s_{1 / 2}}^{\dagger} a_{s_{1 / 2}}^{\dagger}\right]\right\}_{=1, r_{=0}}^{(20)}\right]^{(00)}|0\rangle,
\end{aligned}
$$

where the $K\left(j_{1}, j_{2}\right)$ are state normalization coefficients (in the notation of ref. [8]) and the superscripts give the $\mathrm{SU}(3)$ color quantum numbers. The configuration mixing is very strong, with no amplitudes $\left|c\left(j_{1}, j_{2}\right)\right|>0.300$. Some of the largest cocfficients are, for $m=0$ (with $m=300 \mathrm{MeV}$ in parentheses $), c\left(\mathrm{~s}_{1 / 2}, \mathrm{~s}_{1 / 2}\right)=0.279(0.209), c\left(\mathrm{~s}_{1 / 2}, \mathrm{p}_{3 / 2}\right)=$ $0.300(0.255), c\left(\mathrm{p}_{3 / 2}, \mathrm{p}_{3 / 2}\right) 0.139(0.134), c\left(\mathrm{~s}_{1 / 2}\right.$, $\left.\mathrm{d}_{\mathrm{s} / 2}\right)=0.264(0.231), c\left(\mathrm{~s}_{\mathrm{l} / 2}, \mathrm{f}_{7 / 2}\right)=0.238(0.210)$. Even high-lying quark orbits may have appreciable amplitudes, such as $c\left(f_{7 / 2}, g_{9 / 2}\right)=0.124(0.126)$. Orbits such as $p_{3 / 2}, d_{5 / 2}, f_{7 / 2}, g_{9 / 2}$ may be particularly crucial since the quark density distributions for such orbits have their maxima near $r=R$.

Fig. 2 shows the density distribution of quarks in the six-quark system with a $\left(0 s_{1 / 2}\right)^{2}$ "intrinsic" state coupled to the deuteron quantum numbers $J=1$, $T=0$. Despite the extreme configuration mixing and large excitations into the $0 \mathrm{p}_{3 / 2}, 0 \mathrm{~d}_{5 / 2}, 0 \mathrm{f}_{7 / 2}$ and $0 \mathrm{~g}_{9 / 2}$ orbits, the six-quark density has a maximum at $r=0$. There is not even the beginning of an indication that the quark density distribution shows a spatial separation into two peaks which could be interpreted as separated three-quark clusters.

However, it is interesting to note that the six-quark density is of the desired form if the two quarks coupled to the deuteron quantum numbers $J=1, T=0$

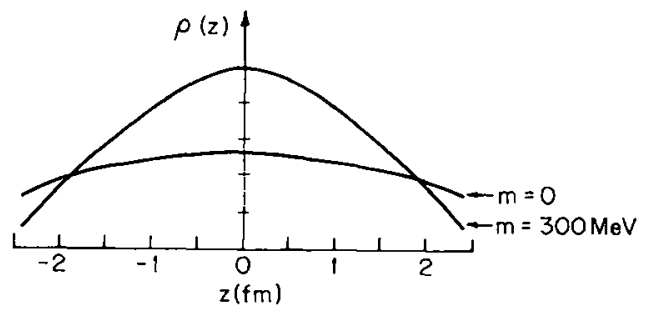

Fig. 2. The quark density distribution for the deuteron. The two quarks not in $J=0, T=0$ coupled pairs are in the $0 s_{1 / 2}$ orbit.

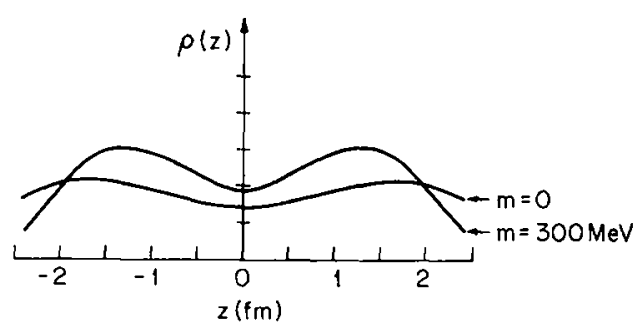

Fig. 3. The quark density distribution for the deuteron, with the two quarks not in $J=0, T=0$ coupled pairs in the $0 \mathrm{p}_{3 / 2}$ orbit.

are promoted from the $0 s_{1 / 2}$ to the $0 p_{3 / 2}$ orbit. Fig. 3 shows that the density distribution of quarks for this case leads to a central minimum with two separated peaks which could be interpreted as the beginning indication of two separated three-quark clusters. For the hamiltonian of eq. (1), however, this would be the density distribution for an excited state at an excitation energy of $206 \mathrm{MeV}(m=0)$ or $139 \mathrm{McV}(m=$ $300 \mathrm{MeV}$ calculation ). For the hamiltonian of eq. (1) there is no mixing between this state and the lower state of fig. 2. However, it has been conjectured [2] that an improved short range quark-quark interaction must also act on the color symmetric SU(3)(20) pair. The necessity for additional interactions has also bcen suggested by Arima et al. [9] in their study of the magnetic moments predicted by the Bonn model. Although a number of interactions have been considered, no two-body quark-quark interaction can reverse the order of the two types of states. The ground state always has a dominant component in which the pair of quarks not coupled to $J=0, T=0$ are in the lowest $0 s_{1 / 2}$ quark state. Such a six-quark state always has a density distribution with a central maximum.

Although the situation therefore appears to be quite different from that in ${ }^{8} \mathrm{Be}$, it should be noted that the "shell model" density for ${ }^{8} \mathrm{Be}$, shown in fig. 1 , is that for an intrinsic state. After projection of the $L=0$ component this shell model density for a purc $s^{4} p^{4}$ configuration would also show a central peak. A central dip again would require very strong configuration mixing of the type predicted, e.g., by the $\operatorname{Sp}(2$, $\mathbb{R})$ model of core excitations in this nucleus. Such calculations indicate that the $\mathrm{s}^{4} \mathrm{p}^{4}$ configuration constitutes only about $30 \%$ of a realistic ${ }^{8} \mathrm{Be}$ shell model wave function [10]. Since we expect the deuteron to be a system of two well separated nucleons it was our 
hope to find an indication of a central minimum in the quark density distribution of this system. Because of the limitations of the arguments based on the one-body density, a more detailed examination of the possible clustering into three-quark clusters will be part of a future study.

In vicw of the successes of the Bonn shell model based on quarks, and in view of the nearly closepacked character of three-quark bags within a nucleus of realistic density, we do not completely rulc out this model as a possible alternate description of the $A$-nucleon system. We do conclude, however, that the Bonn model in its present form is missing a basic ingredient which must be added to furnish the needed strong spatial corrclation into threc-quark clusters. Whether such correlations can be gained from bona fide three-body effects involving three-gluon couplings, or whether such correlations are related to the quark confinement mechanism, would at this stage be shecr speculation.

\section{References}

[1] K. Bleuler, H. Hofestädt, S. Merk and H.R. Petry, Z Naturforsch. 38a (1983) 705; H.R. Petry, Lecture Notes in Physics, Vol. 197 (Springer, Berlin, 1983) p. 236.

[2] H.R. Petry, H. Hofestädt, S. Merk, K. Bleuler, H. Bohr and K.S. Narain, Phys. Lett. B 159 (1985) 363.

[3] H. Hofestädt, S. Merk and H.R. Petry, Z. Phys. A 326 (1987) 391.

[4] Y. Fujiwara, H. Horiuchi, K. Ikeda, M. Kamimura, K. Kato, Y. Suzuki and E. Uegaki, Prog. Theor. Phys. Suppl, 68 (1980) 29.

[5] I. Talmi, Phys. Lett. B 205 (1988) 140.

[6] I. Tonozuka and A. Arima, Nucl. Phys. A 323 (1979) 45

[7] A. Arima, in: Proc. Intern. Conf. on Nuclear physics (Munich, 1973) Vol. 2, p. 192.

[8] K.T. Hecht, Lecture Notes in Physics, Vol. 290 (Springer, Berlin, 1987) p. 95.

[9] A. Arima, K. Yazaki and H. Bohr, Phys. Lett. B 183 (1987) 131

[10] F. Arickx, J. Brocckhove and E. Deumens, Nucl. Phys. A $318(1979) 269$. 